\title{
Universidad de Monterrey ${ }^{1}$
}

\section{Laura Peña-Parás, ${ }^{2}$ Jaime Taha-Tijerina, ${ }^{3}$ Demófilo Maldonado-Cortés ${ }^{4}$}

RESUMEN: Desde 2010, la Universidad de Monterrey (UDEM) cuenta con un nuevo grupo en investigación en nanotecnología y tribología, especializado en las siguientes líneas de investigación: desarrollo y caracterización de nanolubricantes para aplicaciones tribológicas; texturizado de superficies por medio de láser y utilización de nanolubricantes; estudio de nanofluidos para aplicaciones dieléctricas; desarrollo y caracterización de nanofluidos y nanograsas para aplicaciones térmicas; preparación de nanocompuestos poliméricos y pinturas con aditivos de nanopartículas para protección corrosiva y desgaste; nanofluidos como medios de temple avanzados, y, caracterización y funcionalización de nanomateriales con aplicaciones biomédicas.

Se cuenta con personal capacitado y equipo actualizado para hacer frente a las necesidades de la industria y avanzar en temas de ciencia básica y tecnología. La red de contactos científicos e industriales ha crecido considerablemente, lo cual nos permite avanzar en la obtención de recursos para la generación de nuevos y retadores proyectos.Todos los miembros del equipo pertenecen al Sistema Nacional de Investigadores (SNI).

PALABRAS CLAVE: Nanotecnología, nanopartículas, nanolubricantes, nanorrecubrimientos, tribología.

ABSTRACT: Since 2010, the University of Monterrey (UDEM) has a new research group in nanotechnology and tribology, which specializes in the following research areas: development and characterization of nanolubricants for tribological applications; laser surface texturing and application of nanolubricants; study of nanofluids for dielectric applications; development and characterization of nanofluids and nanogreases for thermal applications; preparation of polymer nanocomposites and paints with nanoparticle additives for corrosion and wear protection; nanofluids as advanced quenching medium; characterization and functionalization of nanomaterials with biomedical applications.

Research group has qualified members and totally updated equipment and devices to meet the industrial needs and achieve progress on basic science. The scientific and industrial networking has grown considerably, which allows us to move forward in obtaining resources for the generation of new and challenging projects.

All team members belong to the National Researchers System (SNI).

KEYWORDS: Nanotechnology, nanoparticles, nanolubricants, nanocoatings, tribology.

Recibido: 20 de junio de 2016. Aceptado: 14 de noviembre de 2016.

1 Los autores de este reporte agradecen a la División de Extensión, Consultoría e Investigación (DIECI) de la UDEM, así como a los alumnos que han participado, directa o indirectamente, en los proyectos de investigación y desarrollo de nuestro grupo.

2 Departamento de Ingeniería, Universidad de Monterrey, Ave. Morones Prieto 4500 Pte., San Pedro Garza García, Nuevo León, México, C.P. 66283. Correspondencia: (laura.pena@ udem.edu). Tel. +52 $818215-1000$.

3 Departamento de Ingeniería, UDEM. Correspondencia: (jose.taha@udem.edu).

4 Departamento de Ingeniería, UDEM. Correspondencia: (demofilo.maldonado@udem.edu). 


\section{Breve presentación de la entidad}

En 2010 se abrió la Línea de investigación en "Nanotecnología" Universidad de Monterrey (UDEM), más adelante se obtienen fondos internos para establecer el laboratorio y en 2011 se comenzaron los trabajos de adecuación de espacios y adquisición de equipos. El Laboratorio de Nanotecnología de la UDEM fue inaugurado a principios de 2012, comenzando inmediatamente diversos trabajos de investigación y desarrollo. Este laboratorio fue establecido con el objetivo de realizar proyectos de investigación, consultoría, apoyo a proyectos de tesis de licenciatura y posgrado, así como para la realización de proyectos de cursos y demostraciones de experimentos educativos.

Actualmente se cuenta con 3 profesores-investigadores de tiempo completo que trabajan directamente en el laboratorio para sus proyectos de investigación. Estos académicos forman parte del Sistema Nacional de Investigadores del CONACyT, con la distinción de Investigador Nacional Nivel I. Así también, 4 profesores de la Divisiones de Ingeniería y Tecnologías y de la División de Ciencias de la Salud de la UDEM colaboran con este grupo de manera estrecha en proyectos de investigación básica y aplicada relacionados con nanotecnología formando un equipo multidisciplinario.

El Laboratorio de Nanotecnología trabaja desde sus inicios en conjunto con el Laboratorio de Tribología, principalmente en proyectos relacionados con las líneas de investigación de nanolubricantes y nanorrecubrimientos, siendo este último el que da los resultados cuantitativos en algunas de las investigaciones con las cuales se han obtenido numerosos reconocimientos nacionales e internacionales.

\section{Líneas de investigación en NyN desarrolladas y en curso}

Las líneas principales de investigación del Laboratorio de Nanotecnología se describen a continuación:

- Desarrollo y caracterización de nanolubricantes con propiedades tribológicas superiores de antidesgaste y presiones extremas para utilizarse en la industria metalmecánica en procesos de maquinado y formado (figura 1a).

- Texturizado de superficies con láser y utilización de nanolubricantes para incrementar la resistencia al desgaste de las herramientas de trabajo.

- Estudio de nanofluidos para aplicaciones dieléctricas.

- Desarrollo y caracterización de nanofluidos y nanograsas para aplicaciones térmicas, principalement fluidos de enfriamiento.

- Preparación de nanocompuestos poliméricos y pinturas con aditivos de nanopartículas para protección de superficies metálicas contra corrosión y desgaste (figura $1 \mathrm{~b}$ ). 
FIGURA1. Ejemplos de productos desarrollados en el Laboratorio de Nanotecnología: a) nanolubricantes que disminuyen el desgaste; b) pinturas con aditivos de nanopartículas que incrementan la resistencia a la corrosión.

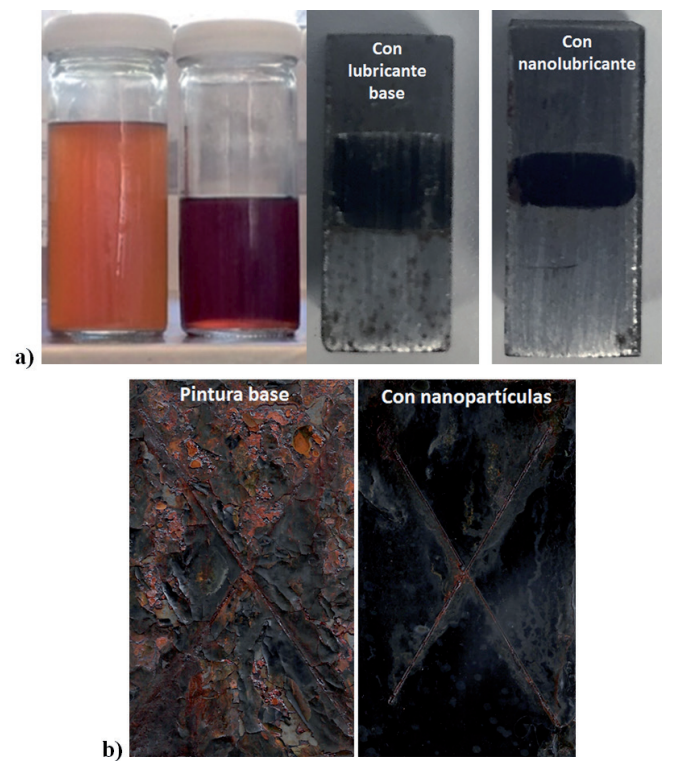

- Nanofluidos como medios de temple avanzados.

- Caracterización y funcionalización de nanomateriales con aplicaciones biomédicas tales como detección de enfermedades y transporte de fármacos.

El grupo de investigación de nanomateriales participa activamente en congresos nacionales e internacionales, entre los cuales destacan, en los últimos 3 años, los listados a continuación:

- $\quad$ STLE 2016 Annual Meeting and Exhibition (Las Vegas, NV, USA) Mayo 2016.

- $\quad$ TMS 2016 (Nashville, TN, USA) - Febrero 2016.

- 20th International Colloquium Tribology (Stuttgart, Alemania) Enero 2016.

- XXXVIII Congreso Nacional de Ingeniería Biomédica 2015 (Mazatlán, SIN, MX) - Octubre 2016.

- STLE Tribology Frontiers Conference 2015 (Denver, CO, USA) - Octubre 2015.

- $\quad$ MS\&T 2015 (Columbus, OH, USA) - Octubre 2015.

- $\quad$ SAMPE 2015 (Baltimore, MD, USA) - Mayo 2015.

- STLE 2015 Annual Meeting and Exhibition (Dallas, TX, USA) Mayo 2015. 
- $\quad$ Wear of Materials (WOM) 2015 (Toronto, CA) - Abril 2015.

- $\quad$ STLE Tribology Frontiers Conference 2014 (Chicago, IL, USA) - Octubre 2014.

- $\quad$ MS\&T 2014 (Pittsburg, PA, USA) - Octubre 2015.

- $\quad$ LUBMAT 2014 (Manchester, UK) - Junio 2014.

- $\quad$ NANOSMAT-USA 2014 (Houston, TX, USA) - Diciembre 2013.

- $\quad$ MS\&T 2013 (Quebec, CA) - Octubre 2013.

- Wear of Materials (WOM) 2013 (Portland, OR, USA) - Abril 2013.

\section{Colaboraciones y proyectos}

El grupo de investigación de nanomateriales del Laboratorio de Nanotecnología colabora estrechamente con diversas instituciones educativas, centros de investigación, así como con la industria metalmecánica regional. En cuanto a colaboraciones internacionales destacan los proyectos realizados con el Institute for Sustainable Technologies - National Research Institute ubicado en Radom, Polonia, en temas de tribología.

Otros proyectos de colaboración en Estados Unidos han sido realizadas con Rice University, de Houston, Texas y con la University of California, de Merced, California. Aunado a esto somos parte de una AERI CONACyT (Alianzas Estratégicas y Redes de Innovación para la competitividad en el tema de conformado de aceros especiales en conjunto con la empresa METALSA).

Hemos trabajado con fondos externos como los otorgados por CONACyT mediante UC MEXUS y los PEI (Programas de Estímulos a la Innovación) y de manera interna con fondos de la División de Investigación Consultoría y Extensión.

Además, actualmente se trabaja con la STLE (Society of Tribologist and Lubrication Engineers) para colaborar con la industria local en abrir el primer capítulo profesional en la región, principalmente en el desarrollo de nanolubricantes.

\section{Infraestructura disponible}

Tal como se mencionó anteriormente, el Laboratorio de Nanotecnología colabora estrechamente con el Laboratorio de Tribología, debido a las diversas líneas de investigación que se trabajan en conjunto sobre preparación y caracterización de nanomateriales. Ejemplos de los equipos de estos laboratorios se muestran en las figuras 2 y 3 .

Para la preparación de nanomateriales se cuenta con los siguientes equipos básicos:

- Campanas de extracción para manejo de químicos y material diverso.

- Gabinete de almacenamiento de químicos. 
- Microbalanzas.

- Medidor de $\mathrm{pH}$.

- Sonicador de punta.

- Sonicadores por inmersión en agua.

- Homogenizadores.

- Planchas de calentamiento y agitación magnética.

- Material de vidrio.

También se tiene el siguiente equipo de de caracterización de materiales:

- Microscopio electrónico de barrido.

- Microscopio óptico.

- Microscopio óptico de medición superficial en 3D.

- Espectrómetro para análisis de metales.

Figura 2. Equipamiento del Laboratorio de Nanotecnología de la Universidad de Monterrey.

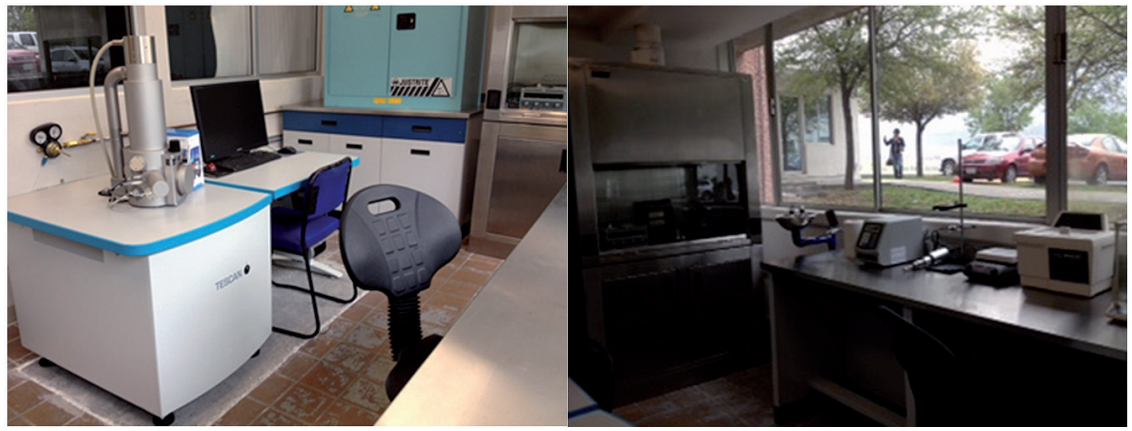

En cuanto a las máquinas para pruebas tribológicas existen los siguientes equipos:

- Máquina para pruebas tribológicas Block-on-ring con capacidad de controlar hasta 6 variables.

- Máquina para pruebas tribológicas Pin-on-Disc, con capacidad de controlar hasta 6 variables.

- Máquina para pruebas tribológicas "4-bolas" con capacidad de realizar 7 pruebas estandarizadas y con control de variables.

- Máquinas tribológicas diseñadas y construidas en la UDEM: "Desgaste abrasivo" y "block on ring para cargas altas".

\section{Docencia y formación de recursos humanos}

Los académicos que forman parte del grupo de investigación en nanomateriales participan activamente en actividades de docencia, tales como la 
Figura 3. Tribotesters del Laboratorio de Tribología de la Universidad de Monterrey y equipos de caracterización de materiales: a) Pin-on-disk; b) Block-on-ring; c) Four-ball; d) espectrómetro; e) microscopio óptico de medición superficial en 3D; f) microscopio óptico.

a)
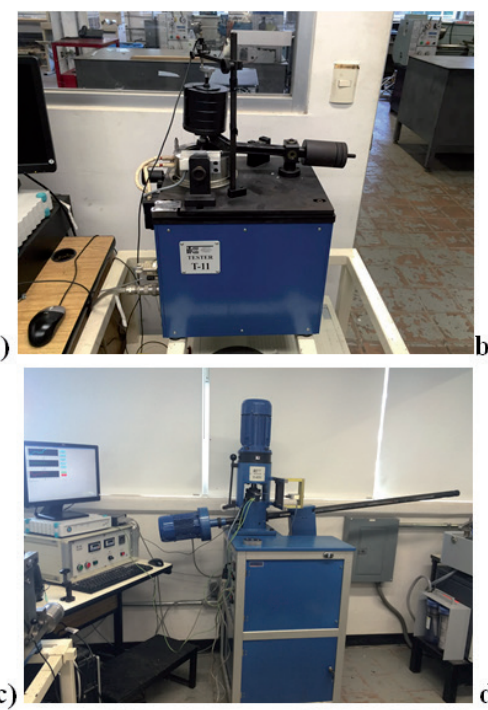

)

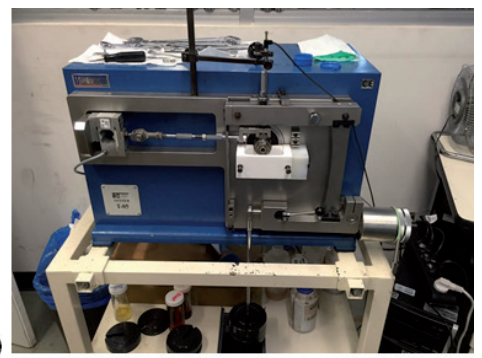

d)
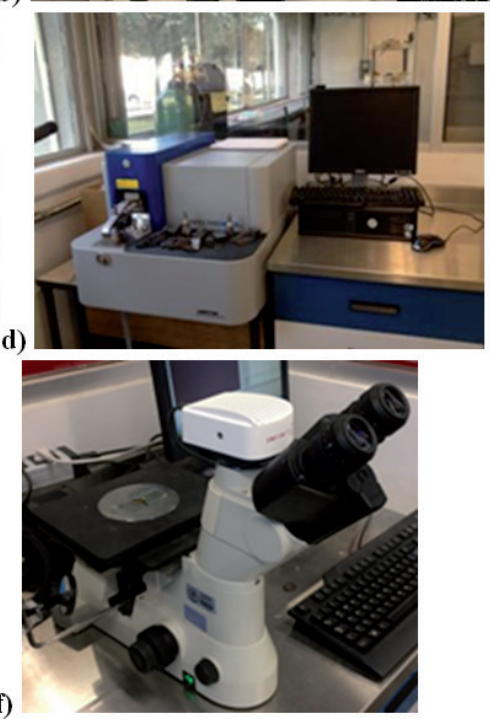

impartición de los cursos de especialidad y electivas de programas de licenciatura y posgrado "Materiales compuestos y nanotecnología" y "Tribología" (con enfoque en nanomateriales) en distintos programas de ingeniería. Estos catedráticos son también responsables de la formación de recursos humanos asesorando proyectos de tesis sobre nanotecnología en programas de licenciatura y posgrado. Gracias a la fundación del Laboratorio de Nanotecnología el número de tesis concluidas en este tema ha crecido considerablemente (figura 4), teniendo un total de 18 tesis de licenciatura de enero de 2011 a la primavera de 2016. Hoy en día hay al menos 3 tesis de maestría en curso.

Los docentes involucrados en este grupo están continuamente capacitándose en temas relacionados con sus líneas de investigación básica y aplicada. 
FIGURA 4. Incremento en el número de tesis de licenciatura concluidas relacionadas con nanotecnología.

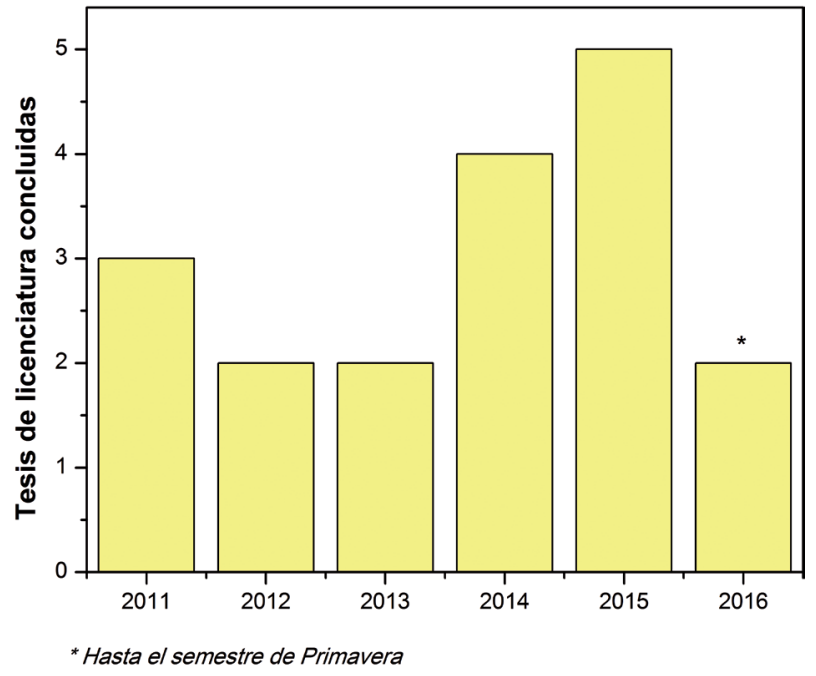

\section{Principales logros}

Los principales logros del grupo de investigación de nanomateriales gracias al Laboratorio de Nanotecnología se describen a continuación:

- La formación de grupos de investigación multidisciplinarios internos, así como con investigadores de instituciones y centros de investigación nacionales e internacionales. Gracias también a este laboratorio se establecieron las líneas de investigación inicialmente de "Nanotecnología" y "Biomateriales" de manera general; a estas líneas se han sumado otras más en años recientes.

- Los desarrollos logrados por el grupo de investigación en nanofluidos y nanolubricantes, para la industria metalmecánica. En recientes estudios ha logrado desarrollar lubricantes con excelentes propiedades de resistencia al desgaste gracias a la adición de nanopartículas. La industria metalmecánica en general cuenta con muchos procesos bajo condiciones de fricción, la cual puede generar un desgaste en la herramienta de trabajo, afectando la calidad final del producto por medio de imperfecciones y fallas; aunado a esto, la fricción resulta en importantes pérdidas energéticas. Los nanolubricantes y nanograsas innovadoras donde nanopartículas son incorporadas como aditivos de alto desempeño, teniendo como objetivo minimizar la fricción, el desgaste, ahorrar en energía y obtener una menor generación de emisiones contaminantes. Asimismo, los nanoaditivos estudiados 
recientemente son biocompatibles y amigables con el ambiente, con el fin de evitar efectos adversos. Actualmente se trabaja con empresas locales para llevar los resultados de laboratorio a la industria.

- En la línea de investigación de pinturas reforzadas con nanopartículas para protección de superficies metálicas se han obtenido resultados de resistencia a la corrosión y desgaste muy superiores a los materiales base, esto también utilizando nanoaditivos ecológicamente amigables.

- Los resultados obtenidos han sido de gran impacto científico y tecnológico; gracias a ellos se han obtenido numerosas distinciones y premios por parte del equipo de trabajo. Entre éstas destacan la obtención de la distinción como Investigador Nacional Nivel I del Sistema Nacional de Investigadores. Entre los premios internacionales se encuentran el Rice University (Materials Science and NanoEngineering Department) "Best Thesis Award". Asimismo, gracias al desarrollo de nanolubricantes se obtuvo el premio otorgado por el MIT Technology Review "Innovadores menores de 35 México" en el 2013. Entre los premios internos se encuentran el "Premio Pro Magistro Roberto Garza Sada 2015", "Premio de Investigación Vinculada a la Docencia" en al menos 5 ocasiones por los trabajos desarrollados en este laboratorio, así como el "Premio al Investigador UDEM".

- En cuanto a producción científica de manera periódica publicamos artículos en revistas indexadas, se ha aceptado para su publicación un capítulo de libro titulado "Thermal and tribological characterization of 2D-based nanofluids" en el libro 2D Materials, y uno de los integrantes del grupo es autor del libro Tribotesting: Reproducibility and repeatability problems, publicado en 2010.

- Gracias también al reconocimiento internacional por nuestros trabajos en nanolubricantes, el grupo de investigación logró acreditar el primer capítulo estudiantil de la STLE en México (Society of Tribologist and Lubrication Engineers) con sede en la UDEM.

La figura 5 muestra el incremento en la cantidad de productos generados, como artículos, ponencias, tesis dirigidas y premio obtenidos por el grupo de investigación.

\section{Retos a futuro}

El desarrollo de nanomateriales presenta los siguientes grandes retos que el grupo trabaja para resolver:

- Utilización de nanopartículas biocompatibles y por ende amigables con el ambiente: en este sentido hemos iniciado con éxito el uso de nanoarcillas que han demostrado tener excelentes propiedades antidesgaste y anticorrosión además de no ser tóxicas. 
FIGURA 5. Incremento en productos generados de enero de 2011 a mayo de 2016.

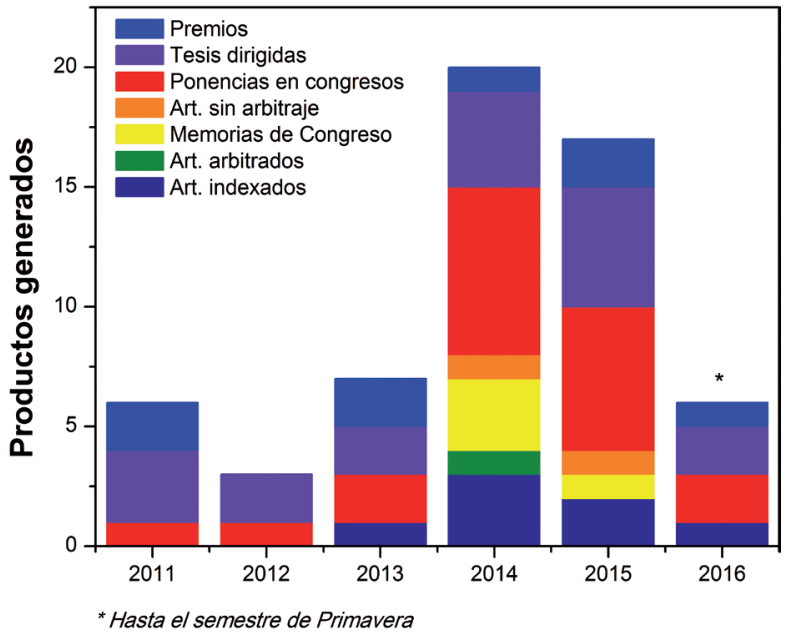

- Lograr mantener la óptima dispersión y cantidad de las nanopartículas con el objetivo de no perder propiedades; esto lo hemos ido logrando mediante el manejo de variables como porcentaje de peso añadido, tipo de nanopartícula, tamaño y en agentes dispersantes.

- En el caso de nanolubricantes, comprender los efectos del acabado superficial y características geométricas de las nanopartículas en el efecto antidesgaste en paralelo con el estudio de los mecanismos de fricción y regímenes de lubricación.

\section{Espacios web institucionales relacionados con NyN}

- Divisón de Investigación, Consultoría y Extensión de la UDEM: $<$ http://www.udem.edu.mx/Esp/Investigacion-y-Desarrollo/Pages/ objetivo.aspx>.

- Portafolio de Investigadores y Consultores de la UDEM: <http:// www.udem.edu.mx/Esp/Investigacion-y-Desarrollo/Pages/investigadores-y-consultores.aspx $>$.

- CV de la Dra. Laura Peña: <http://www.udem.edu.mx/Esp/Profesores/Pages/detalles.aspx?Division=DIT\&Departamento=Ingenier\% C3\%ADa\&IdProfesor=322>.

- CV del Dr. Jaime Taha: <http://www.udem.edu.mx/Esp/Profesores/Pages/detalles.aspx?Division=DIT\&IdProfesor $=386\rangle$. 